\section{Relação entre a cobertura da Estratégia Saúde da Família e o diagnóstico de sífilis na gestação e sífilis congênita}

\author{
Coverage by the Family Health Strategy \\ and diagnosis of syphilis in pregnancy \\ and congenital syphilis
}

Valéria Saraceni 1

Angélica Espinosa Miranda 2

\footnotetext{
1 Secretaria Municipal de Saúde e Defesa Civil, Prefeitura da Cidade do Rio de Janeiro, Rio de Janeiro, Brasil.

2 Núcleo de Doenças Infecciosas, Universidade Federal do Espírito Santo, Vitória, Brasil.

\section{Correspondência}

V. Saraceni

Superintendência de Vigilância em Saúde, Secretaria Municipal de Saúde e Defesa Civil, Prefeitura da Cidade do Rio de Janeiro.

Rua Cupertino Durão 219, bloco B, apto. 404, Rio de Janeiro, RJ 22441-030, Brasil. valsaraceni@gmail.com
}

\begin{abstract}
This paper aimed to correlate syphilis in pregnancy and congenital syphilis with coverage of the Family Health Strategy (FHS), based on available data in the national health information systems. The syphilis notification estimates were calculated according to the Sentinel Childbirth Study for 2004 under the Ministry of Health and the data were obtained from the websites of the Health Surveillance Secretariat and Healthcare Secretariat, for the year 2008. The ratios between observed and estimated gestational syphilis and congenital syphilis were not statistically correlated with population coverage by the FHS $(r=-0.28$ and $r=-0.40$, respectively). The FHS is a privileged area for prenatal care and logically a source of compulsory notification of syphilis in pregnancy. By combining diagnosis with adequate treatment of syphilis in pregnant women and their partners, the FHS becomes a prime instrument for eliminating congenital syphilis in Brazil. Expanding the FHS coverage and quality of care are essential for achieving this goal.

Congenital Syphilis; Pregnancy; Family Health; Syphilis
\end{abstract}

\section{Introdução}

A notificação compulsória de uma doença visa acumular dados suficientes para permitir uma análise que leve a intervenções para sua redução e/ou de suas consequências ${ }^{1}$. No caso da notificação da sífilis em gestantes, a pretensão clara é a de reduzir a sua incidência até conseguir a eliminação da sífilis congênita, derivada da transmissão vertical do Treponema pallidum ${ }^{2}$. A sífilis congênita é causa de importante morbidade para a criança e, também, de mortalidade perinatal, especialmente no componente fetal 3 , apesar do baixo custo do tratamento e disponibilidade de tecnologia leve para sua prevenção 4 .

A sífilis na gestante tornou-se de notificação compulsória em 2005 (Portaria MS/SVS nº. 33) 5, sendo a ficha de investigação liberada para digitação no Sistema Nacional de Agravos de Notificação (SINAN) em 2007. A sífilis congênita adquiriu este status muito tempo antes, em 1986, e sua ficha de investigação sofreu variações ao longo dos anos, a fim de melhorar a detecção e acompanhar o processo de eliminação assinado em acordos internacionais pelo Ministério da Saúde 6,7,8.

A qualidade do preenchimento das fichas de investigação de agravos de notificação compulsória é determinante para o planejamento de intervenções 1 . Para a sífilis em gestante, o processo de investigação se dá na atenção primária, uma vez que esse é o cenário em que o diagnóstico 
da doença e a assistência pré-natal se desenvolvem. Portanto, a atenção primária é o momento de ocorrência da notificação e investigação do caso. Da mesma forma que é o momento em que o cuidado pré-natal deve agir e evitar o caso de sífilis congênita ${ }^{9}$. A atenção primária se expande pela Estratégia Saúde da Família (FHS), preconizada pelo Ministério da Saúde 10. Giovanella et al. 11 relataram a presença da FHS em $94 \%$ dos municípios brasileiros em 2008, correspondendo a uma cobertura populacional de $48 \%$. Nessa estratégia se insere a atenção pré-natal, a qual tem em seus protocolos a triagem da sífilis na grávida e o consequente tratamento da gestante e de seu parceiro, quando apresentam testes positivos 9 .

O Ministério da Saúde estimou a prevalência de sífilis em gestantes no Brasil com o Estudo Sentinela Parturiente, de 2004 12. As estimativas foram feitas para o nível das macrorregiões e, a partir dessas estimativas, a Secretaria de Vigilância em Saúde do Ministério da Saúde (SVS/MS) vem trabalhando com o número esperado de casos, aplicando a prevalência estimada ao número de nascidos vivos. O número de casos de sífilis em gestante e o número de casos de sífilis congênita são indicadores da Pactuação de Ações da Vigilância em Saúde (PAVS) e do Pacto pela Vida, Saúde e Gestão (SISPACTO) 13,14. Considerando uma transmissão vertical da sífilis de $25 \%$, é possível estimar o número de casos esperados de sífilis congênita para cada região do país. A inclusão desses dois indicadores nas pactuações que envolvem as três FHSeras do Sistema Único de Saúde (SUS) demonstra claramente a vontade política do Ministério da Saúde em atingir a eliminação da sífilis congênita, ou seja, 0,5 casos de sífilis congênita por mil nascidos vivos 4 .

Partindo-se, então, da premissa de que a expansão da atenção básica levará a um aumento da cobertura pré-natal e, consequentemente, da cobertura dos exames de triagem no pré-natal, pretendeu-se com este trabalho investigar a relação entre a cobertura da FHS nas regiões brasileiras e a razão de casos de sífilis em gestante e de sífilis congênita observados entre os casos estimados, para o ano de 2008.

\section{Metodologia}

Os casos observados dos dois agravos em tela foram obtidos no SINANNET disponível na Internet, na página da SVS/MS, por macrorregião do Brasil para o ano de 2008. Esse ano foi escolhido pela maior estabilidade dos números e por ser o ano com dados mais recentes (SVS/ MS; http://dtr2004.saude.gov.br/sinanweb/ index.php?saude, acessado em 10/Mai/2011). O número de nascidos vivos foi retirado da página do Departamento de Informática do SUS (DATASUS; http://www2.datasus.gov.br/ DATASUS/index.php?area=0205\&VObj=http: / / tabnet.datasus.gov.br/cgi/deftohtm.exe?sinasc/ cnv/nv, acessado em 10/Mai/2011). Foram calculados os números estimados de casos de sífilis em gestante e de sífilis congênita por região brasileira, de acordo com a prevalência encontrada no estudo sentinela parturiente 2004 12. Foram calculadas então as razões de casos observados sobre os estimados para os dois agravos. A cobertura de FHS foi obtida na página do Departamento de Atenção Básica do Ministério da Saúde (http://dab.saude.gov.br/histórico_cobertu ra_sf.php, acessado em 10/Mai/2011), por competências e por região para o mês de dezembro de 2008. Aplicou-se o coeficiente de correlação de Pearson para verificar a ocorrência de uma possível relação entre a cobertura de FHS e as razões de casos encontrados entre os estimados para as duas doenças por macrorregião.

O presente estudo foi realizado com dados secundários disponibilizados pelo Ministério da Saúde na Internet, garantindo o anonimato dos indivíduos, em conformidade com a Resolução $n^{o}$. 196/96 do Conselho Nacional de Saúde.

\section{Resultados}

Existem grandes diferenças regionais quanto ao número de nascidos vivos, mas a prevalência de sífilis na gestação gira em torno da média nacional, que foi estimada em 1,6\% 12. A cobertura populacional pela FHS variou bastante entre as regiões, sendo mais alta na Região Nordeste (71\%) e mais baixa na Região Sudeste (37\%). O número de nascidos vivos em 2008, a prevalência estimada pelo estudo sentinela para sífilis em gestantes e a cobertura de FHS em dezembro de 2008 por região podem ser vistos na Tabela 1 .

Os casos observados e estimados de sífilis em gestante, a razão observada/estimada para esse agravo, os casos encontrados e estimados de sífilis congênita e a razão observada/estimada para sífilis congênita por região estão na Tabela 2. A razão observada/estimada para sífilis na gestação variou de 0,10 a 0,38 , enquanto a razão observada/estimada de sífilis congênita variou de 0.41 a 0,66 entre as regiões.

A Figura 1 mostra a distribuição da razão observada/estimada de sífilis em gestante e da cobertura populacional de FHS para as 5 macrorregiões. O coeficiente de correlação de Pearson resultou em $r=-0,28(p=0,646)$, denotando uma ausência de associação. A Região Centro-oeste 
Número de nascidos vivos, prevalência estimada pelo estudo sentinela para sífilis em gestantes e cobertura da Estratégia Saúde da Família (FHS) por macrorregião brasileira, 2008.

\begin{tabular}{lccc}
\hline Macrorregião & Nascidos vivos & Sífilis na gestação (\%) & Cobertura de FHS (\%) \\
\hline Norte & 321.998 & 1,8 & 47 \\
Nordeste & 888.268 & 1,9 & 71 \\
Sudeste & 1.130 .407 & 1,6 & 37 \\
Sul & 371.497 & 1,4 & 48 \\
Centro-Oeste & 222.658 & 1,3 & 48
\end{tabular}

Fonte: Sistema de Informações sobre Nascidos Vivos (Departamento de Informática do SUS, 2008); Estudo Sentinela Parturiente de 2004 (Departamento de Atenção Básica, Ministério da Saúde, 2008).

Tabela 2

Casos observados e estimados e razão observada/estimada para sífilis em gestante, casos observados e estimados de sífilis congênita e razão observada/estimada para sífilis congênita, por macrorregião brasileira, 2008.

\begin{tabular}{|c|c|c|c|c|c|c|}
\hline \multirow[t]{2}{*}{ Macrorregião } & \multicolumn{3}{|c|}{ Sífilis em gestantes } & \multicolumn{3}{|c|}{ Sífilis congênita } \\
\hline & $\begin{array}{c}\text { Casos } \\
\text { observados }\end{array}$ & $\begin{array}{c}\text { Casos } \\
\text { estimados }\end{array}$ & $\begin{array}{c}\text { Razão } \\
\text { observada/ } \\
\text { estimada }\end{array}$ & $\begin{array}{c}\text { Casos } \\
\text { observados }\end{array}$ & $\begin{array}{c}\text { Casos } \\
\text { estimados }\end{array}$ & $\begin{array}{c}\text { Razão } \\
\text { observada/ } \\
\text { estimada }\end{array}$ \\
\hline Norte & 1.256 & 5.796 & 0,22 & 957 & 1.449 & 0.66 \\
\hline Nordeste & 1.751 & 16.877 & 0,10 & 1.926 & 4.219 & 0.46 \\
\hline Sudeste & 2.445 & 18.087 & 0,14 & 2.502 & 4.522 & 0.55 \\
\hline Sul & 692 & 5.201 & 0,13 & 535 & 1.300 & 0.41 \\
\hline Centro-oeste & 1.109 & 2.895 & 0,38 & 433 & 724 & 0.60 \\
\hline
\end{tabular}

Fonte: Sistema Nacional de Agravos de Notificação (Secretaria de Vigilância em Saúde, Ministério da Saúde, 2008); Estudo Sentinela Parturiente de 2004 (Departamento de Atenção Básica, Ministério da Saúde, 2008).

foi a que apresentou a maior razão observada/ estimada $(0,38)$ para sífilis na gestação, embora não apresentasse a maior proporção de cobertura de FHS (60\%). A Região Nordeste apresentou a maior cobertura de FHS (71\%), porém com uma baixa razão observada/estimada $(10 \%)$. As regiões Norte, Sudeste e Sul apresentaram uma razão observada/estimada para sífilis na gravidez menor do que 0,25 e, também, uma baixa cobertura de FHS, menor do que $50 \%$.

Em relação à razão observada/estimada de sífilis congênita e a cobertura de FHS, a correlação também não foi significativa (coeficiente de correlação de Pearson, $r=-0,40, p=0,507$ ), com a razão observada/estimada de sífilis congênita não atingindo $70 \%$ na Região Norte, que foi a região que apresentou o maior valor $(0,66)$, seguida da Região Centro-Oeste $(0,60)$, como pode ser conferido na Figura 2. Correlacionado as razões observadas/estimadas de sífilis em gestante e sífilis congênita, e partindo do pressuposto que ambas as detecções se encontravam aquém do esperado, encontramos um valor do coeficiente de correlação de Pearson de 0.64.

\section{Discussão}

Com base na estimativa derivada de uma metodologia com poder para demonstrar a prevalência de casos de sífilis em gestante nas macrorregiões brasileiras 12, encontrou-se uma razão de casos observados sobre casos estimados muita baixa. Da mesma forma, utilizando-se uma taxa de transmissão vertical de $25 \%$ para a sífilis, a razão de casos observados sobre casos estimados de sífilis congênita também ficou aquém do esperado. Vários estudos no Brasil têm mostrado a persistência da sífilis na gravidez e da sífilis congênita $15,16,17,18,19$. 
Figura 1

Distribuição da razão observada/estimada de sífilis em gestante pela cobertura populacional da Estratégia Saúde da Família (FHS) por macrorregião brasileira, 2008.

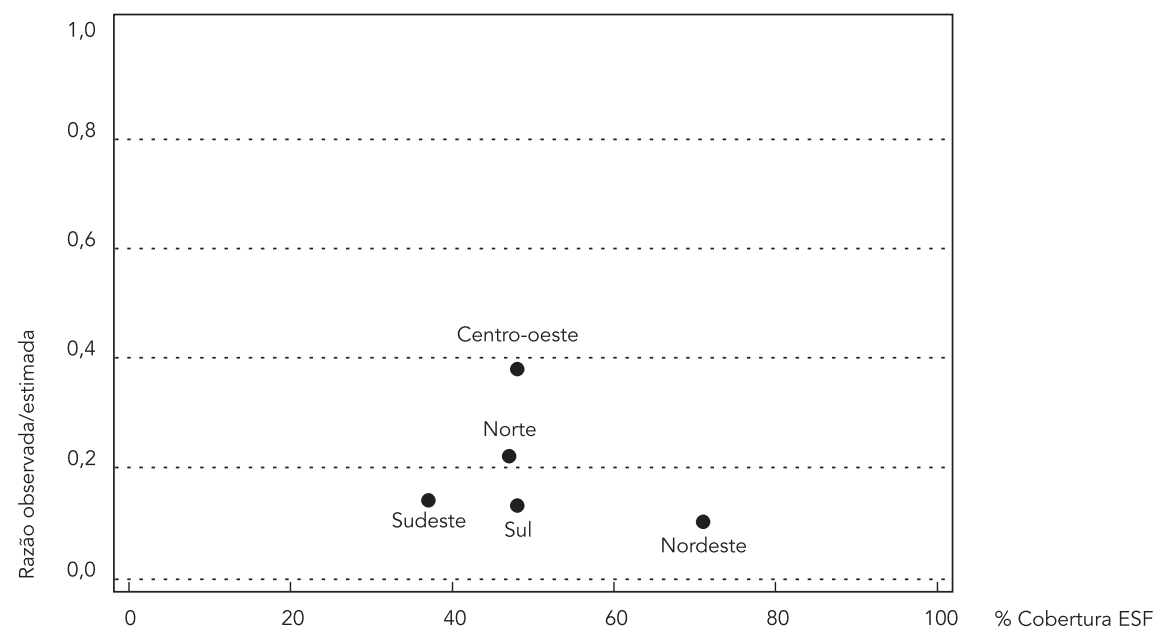

Fonte: Sistema Nacional de Agravos de Notificação (Secretaria de Vigilância em Saúde, Ministério da Saúde, 2008); Estudo Sentinela Parturiente de 2004 (Departamento de Atenção Básica, Ministério da Saúde, 2008).

Figura 2

Distribuição da razão observada/estimada de sífilis congênita pela cobertura populacional da Estratégia Saúde da Família (FHS) por macrorregião brasileira, 2008

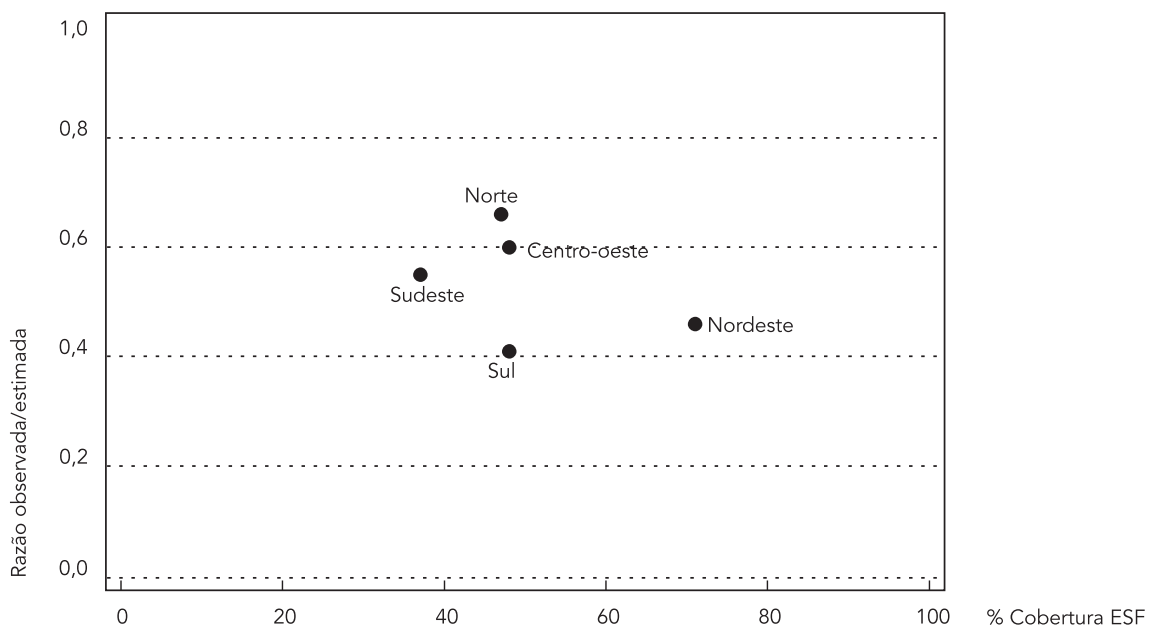

Fonte: Sistema Nacional de Agravos de Notificação (Secretaria de Vigilância em Saúde, Ministério da Saúde, 2008); Estudo Sentinela Parturiente de 2004 (Departamento de Atenção Básica, Ministério da Saúde, 2008). 
A triagem bem realizada no pré-natal, seguida do tratamento adequado da gestante e parceiro(s), deveria apontar para uma razão de casos observados sobre casos estimados de sífilis na gestação próxima de 1,0, independentemente da cobertura da FHS, pois é sabido que nem todas as ações de assistência pré-natal são executadas na atenção básica. Entretanto, optou-se por utilizar a cobertura de FHS, que se encontra em expansão, como um proxy do aumento do acesso ao cuidado (http://dab.saude.gov.br/histórico_ cobertura_sf.php, acessado em 10/Mai/2011). Na sua pesquisa sobre limites e possibilidades da atenção primária à saúde em quatro capitais brasileiras, Giovanella et al. ${ }^{11}$ relataram ser a FHS a porta de entrada preferencial de acesso à saúde, tanto por parte dos gestores, como dos médicos e enfermeiros entrevistados.

A razão observada/estimada mais alta encontrada neste estudo não chegou a 0,40 para a sífilis na gestante. Tal fato imediatamente gera a inferência de que, se não foram encontradas e tratadas todas as gestantes infectadas e seus parceiros, obviamente ainda serão encontrados muitos casos de sífilis congênita. Esse foi o cenário para o ano de 2008, aqui analisado. A razão mais alta para sífilis congênita, encontrada na Região Norte permite imaginar que em função das enormes distâncias regionais, muitos casos de sífilis na gestação não sejam diagnosticados, permitindo a ocorrência de muitos casos de sífilis congênita 15.

No momento em que a sífilis na gestação for um agravo controlado e a razão observada/estimada se aproximar de 1,0, teremos, então, uma diminuição real do número de casos de sífilis congênita, e a razão para sífilis congênita se aproximará do zero, refletindo a meta de eliminação, ou seja, uma incidência menor do que 0,5 casos por mil nascidos vivos ${ }^{4}$. Não se pode esquecer que sempre existirá a possibilidade de falha de tratamento 20 ou da ocorrência de um caso em que a gestante não realizou o pré-natal. Cada vez menos se deve encontrar o nome de uma gestante com sífilis notificada no SINAN repetido como a mãe de um caso de sífilis congênita notificado ao mesmo sistema de informação, quando do relacionamento dessas duas diferentes bases de dados (sífilis em gestante e sífilis congênita), como forma de avaliar o que está acontecendo no nível municipal, por exemplo. A cada gestante adequadamente tratada, não haverá um caso de sífilis congênita. Assim, a gestante notificada como caso na base de sífilis em gestante não irá aparecer como a mãe de um caso de sífilis congênita na base de sífilis congênita.

Portanto, mais uma vez se demonstra a relevância do cuidado pré-natal na eliminação da sífilis congênita. A FHS tem, em sua concepção, espaços de integração e capacitação das equipes que devem ser aproveitados para a qualificação do cuidado prestado à população adstrita. O acesso privilegiado à família pode facilitar o diagnóstico e o tratamento do casal, de acordo com o protocolo clínico 21 . Sem falar de que dispõe de mecanismos de busca ativa de casos e de faltosos por intermédio dos agentes comunitários de saúde. Outro aspecto importante a comentar é a realização de pelo menos dois testes de triagem (VDRL) durante o pré-natal, com a consequente anotação clara e precisa dos resultados e do tratamento, se a gestante for positiva. Deve-se anotar se a gestante foi tratada, com que esquema, se foi completado adequadamente, o resultado do parceiro e o tratamento dele, tudo no cartão da gestante, com o qual ela dará entrada na maternidade. No estudo sentinela parturiente realizado em 2006, apenas 16,9\% das parturientes entrevistadas informaram ter o cartão consigo e os dois testes anotados 22. Essa é mais uma ação que pode ser bem realizada no âmbito da FHS. Contudo, para a eliminação da sífilis congênita é preciso atentar para os vários componentes da ação programática de pré-natal 23 , pois um atendimento pré-natal parcialmente implantado na FHS, em que nem todas as atividades preconizadas em protocolos estão sendo aplicadas, pode ser um fator negativo para o sucesso. Nesse território podem ocorrer mais casos de sífilis congênita do que em outro no qual o pré-natal se encontra implantado 24 .

Na questão da integração da vigilância em saúde e assistência, novamente a FHS se coloca em condição favorável, já que pode efetuar a notificação do agravo uma vez feito o diagnóstico e o tratamento da gestante e do parceiro ${ }^{5}$. A participação dos agentes comunitários de saúde e dos agentes de vigilância em saúde nas discussões das equipes da FHS pode incrementar a identificação de casos de agravos de notificação compulsória em seu território e, com isso, aumentar a cobertura da vigilância em saúde dentro da FHS.

Precisamos mencionar algumas limitações do estudo para que os resultados sejam avaliados com cautela. Número de casos de sífilis na gestação, de sífilis congênita e a cobertura da FHS são dados secundários sem validação independente, provenientes da administração da saúde e passíveis de viés de informação. Outra limitação é oriunda do delineamento de um estudo ecológico, o qual não permite interpretações diretas dos resultados no nível individual. A abordagem descritiva se limita às análises univariadas, não ajustando por diversos fatores de risco e suas interações, nem por estrutura espacial de dados 
o que seria possível numa abordagem analítica mais complexa.

Poderia ser argumentado que se utilizou como fundamento uma informação de base secundária, as notificações ao SINAN, que poderiam não retratar a realidade. Contudo, por ser de notificação compulsória, o SINAN é a fonte de dados de maior amplitude para realizar esta avaliação. E uma vez que os dados foram analisados por macrorregião, a distribuição da possível subnotificação se dilui de forma menos heterogênea. Por ser mais antiga na lista de notificação compulsória, a sífilis congênita pode apresentar uma precedência em relação à sífilis em gestante, com a primeira apresentando maior cobertura. Entretanto, pela participação de ambos os agravos nas pactuações nacionais aqui referidas, que envolvem prestações de conta e financiamento para estados e municípios, é de se esperar uma boa cobertura para os dois.

\section{Conclusão}

A análise dos dados macrorregionais de sífilis em gestante e de sífilis congênita mostra que ainda há um longo caminho a percorrer para a eliminação da sífilis congênita. Ao se acoplar essa análise à cobertura de FHS, procurou-se chamar a atenção para a ampliação do cuidado atrelada à expansão inevitável, que tem tudo para ser o elo que faltava na corrente de eliminação. A FHS se apresenta como o espaço privilegiado para o diagnóstico precoce da sífilis na gestante e a consequente eliminação da sífilis congênita.

\section{Resumo}

Este estudo procurou correlacionar as informações disponíveis em sistemas nacionais de informação em saúde sobre notificações de sífilis em gestante, sífilis congênita e cobertura populacional da Estratégia Saúde da Família (FHS). As estimativas de notificação foram calculadas de acordo com o Estudo Sentinela Parturiente de 2004 do Ministério da Saúde e os dados observados foram obtidos na Internet, nas páginas da Secretaria de Vigilância em Saúde e da Secretaria de Atenção em Saúde, para o ano de 2008. As razões observadas sobre estimadas para sífilis em gestante e sífilis congênita e a cobertura populacional da FHS por macrorregião brasileira não apresentaram correlação ( $r=-0,28$ e $r=-0,40$, respectivamente). A FHS se apresenta como local privilegiado para realização do pré-natal e, logicamente, fonte da notificação compulsória de sífilis em gestante. Acoplando diagnóstico com o tratamento adequado da sífilis na gestante e no parceiro, a FHS é instrumento primordial para a eliminação da sífilis congênita no Brasil. Expansão da cobertura e cuidado de qualidade são essenciais para o alcance da meta.

Sífilis Congênita; Gravidez; Saúde da Família; Sífilis

\section{Colaboradores}

V. Saraceni contribuiu com a concepção, coleta e análise dos dados e redação do artigo. A. E. Miranda contribuiu com a concepção, desenho, redação e revisão do artigo. 


\section{Referências}

1. Laguardia J, Domingues CMA, Carvalho C, Lauerman CR, Macário E, Glatt R. Sistema de Informação de Agravos de Notificação em Saúde (SINAN): desafios no desenvolvimento de um sistema de informação em saúde. Epidemiol Serv Saúde 2004; 13:135-46.

2. Sánchez PJ, Wendel GD. Syphilis in pregnancy. Clin Perinatol 1997; 24:71-90.

3. Saraceni V, Guimarães MH, Theme Filha MM, Leal MC. Mortalidade perinatal por sífilis congênita: indicador da qualidade da atenção à mulher e à criança. Cad Saúde Pública 2005; 21:1244-50.

4. Programa Nacional de DST e AIDS, Secretaria de Assistência à Saúde, Secretaria de Vigilância em Saúde, Ministério da Saúde. Plano operacional redução da transmissão vertical do HIV e da sífilis. Brasília: Ministério da Saúde; 2007.

5. Ministério da Saúde. Portaria MS/SVS no. 33, de 15 de julho de 2005. Diário Oficial da República Federativa do Brasil 2005; 15 jul.

6. Paz LC, Pereira GF, Pinto VM, Medeiros MG, Matida LH, Saraceni V, et al. New case definition of congenital syphilis for epidemiological surveillance purposes in Brazil, 2004. Rev Soc Bras Med Trop 2005; 38:446-7.

7. Tayra A, Matida LH, Saraceni V, Paz LC, Ramos Junior AN. Two decades of epidemiological surveillance of congenital syphilis in Brazil: concerning the case definitions. DST J Bras Doenças Sex Transm 2007; 19:111-9.

8. Valderrama J, Urquia-Bautista A, Orlich G, Siri RS, Luz-Osimani M, Abreu H, et al. Maternal and congenital syphilis: case definitions. Epidemiol Bull 2005; 26:12-5.

9. Departamento de Ações Estratégicas, Secretaria de Atenção à Saúde, Ministério da Saúde. Pré-natal e puerpério - atenção qualificada e humanizada. Brasília: Ministério da Saúde; 2006.

10. Departamento de Atenção Básica, Secretaria de Atenção à Saúde, Ministério da Saúde. Política nacional de atenção básica. Brasília: Ministério da Saúde; 2006.

11. Giovanella L, Mendonça MHM, Almeida PF, Escorel S, Senna MCM, Fausto MCR, et al. Family health: limits and possibilities for an integral primary care approach to health care in Brazil. Ciênc Saúde Coletiva 2009; 14:783-94.

12. Programa Nacional de DST e AIDS, Secretaria de Vigilância em Saúde, Ministério da Saúde. Relatório: primeiros resultados do Estudo-Sentinela Parturiente 2004. http://www.aids.gov.br/data/Pages/ LUMIS286DF0DAPTBRIE.htm (acessado em 11/ Nov/2006).

13. Ministério da Saúde. Portaria no ${ }^{\circ}$ 3008, de 1o de dezembro de 2009. Determina a Programação das Ações de Vigilância em Saúde (PAVS), como um elenco norteador para o alcance de metas do Pacto e demais prioridades de relevância para o Sistema Nacional de Vigilância em Saúde e Vigilância Sanitária, eleitas pelas FHSeras Federal, Estadual e Municipal. Diário Oficial da União 2009; 2 dez.
14. Departamento de Apoio à Gestão Descentralizada, Secretaria Executiva, Ministério da Saúde. Orientações acerca dos indicadores de monitoramento e avaliação do pacto pela saúde, nos componentes pela vida e de gestão para o biênio 2010-2011. Brasília: Ministério da Saúde; 2011.

15. Benzaken AS, García EG, Rodrigues AO, Mora C, Sanchez MS, Sardinha JC. "Madre Fronteriza"; estudo conjunto de prevalência de sífilis tricomoníase e HIV em gestantes da tríplice fronteira do Alto Rio Solimões. DST J Bras Doenças Sex Transm 2004; 16:15-23.

16. Miranda AE, Alves MC, Neto RL, Areal KR, Gerbase AC. Seroprevalence of HIV, hepatitis B virus, and syphilis in women at their first visit to public antenatal clinics in Vitória, Brazil. Sex Transm Dis 2001; 2812:710-3

17. Saraceni V, Leal MC. Avaliação da efetividade das campanhas para eliminação da sífilis congênita na redução da morbimortalidade perinatal: Município do Rio de Janeiro, 1999-2000. Cad Saúde Pública $2003 ; 19: 1341-9$.

18. Figueiró-Filho EA, Senefonte FR, Lopes AH, Morais OO, Souza Júnior VG, Maia TL, et al. Frequency of HIV-1, rubella, syphilis, toxoplasmosis, cytomegalovirus, simple herpes virus, hepatitis B, hepatitis C, Chagas disease and HTLV I/II infection in pregnant women of State of Mato Grosso do Sul. Rev Soc Bras Med Trop 2007; 40:181-7.

19. Madi JM, Souza RS, Araújo BF, Oliveira Filho PF Rombaldi RL, Mitchell C, et al . Prevalence of toxoplasmosis, HIV, syphilis and rubella in a population of puerperal women using Whatman $903 \AA$ filter paper. Braz J Infect Dis 2010; 14:24-9.

20. Alexander JM, Sheffield JS, Sanchez PJ, Mayfield J, Wendel GD. Efficacy of treatment for syphilis in pregnancy. Obstet Gynecol 1999; 93:5-8.

21. Departamento de Atenção Básica, Secretaria de Atenção à Saúde, Ministério da Saúde. Saúde sexual e saúde reprodutiva. Brasília: Ministério da Saúde; 2010.

22. Szwarcwald CL, Barbosa Junior A, Miranda AE Paz LC. Results of the sentinel surveillance project, 2006: challenges for the control of congenital syphilis in Brazil. DST J Bras Doenças Sex Transm 2007; 19:128-33.

23. Saraceni V, Vellozo VRO, Leal MC, Hartz ZMA. Avaliação das campanhas para a eliminação da sífilis congênita, no município do Rio de Janeiro, a partir de um modelo teoricológico. Rev Bras Saúde Matern Infant 2005; 5 Suppl 1:S33-41.

24. Vidal SA, Samico IC, Frias PG, Hartz ZMA. Estudo exploratório de custos e consequências do prénatal no Programa Saúde da Família. Rev Saúde Pública 2011; 45:467-74.

Recebido em 21/Jun/2011

Versão final reapresentada em 24/Out/2011

Aprovado em 21/Nov/2011 